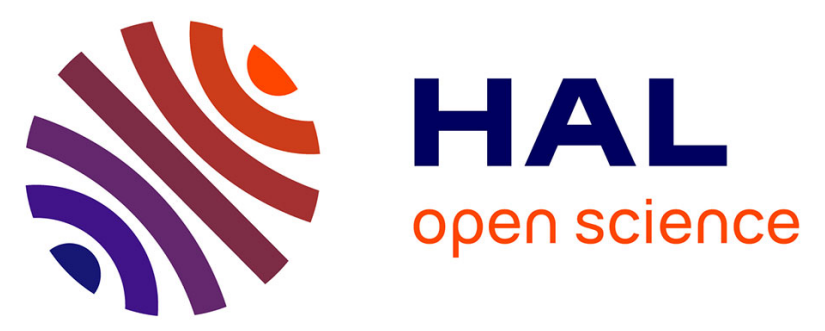

\title{
"Dés-élitiser" la recherche pour favoriser l'équité en santé. Les recherches participatives avec des publics en situation de pauvreté en santé publique
}

Christine Loignon, Sophie Dupéré, Baptiste Godrie, Caroline Leblanc

\section{- To cite this version:}

Christine Loignon, Sophie Dupéré, Baptiste Godrie, Caroline Leblanc. "Dés-élitiser " la recherche pour favoriser l'équité en santé. Les recherches participatives avec des publics en situation de pauvreté en santé publique. Éthique publique - revue internationale d'éthique sociétale et gouvernementale , 2018, vol. 20, n 2, 10.4000/ethiquepublique.4058 . hal-02957204

\author{
HAL Id: hal-02957204 \\ https://hal.science/hal-02957204
}

Submitted on 4 Oct 2020

HAL is a multi-disciplinary open access archive for the deposit and dissemination of scientific research documents, whether they are published or not. The documents may come from teaching and research institutions in France or abroad, or from public or private research centers.
L'archive ouverte pluridisciplinaire HAL, est destinée au dépôt et à la diffusion de documents scientifiques de niveau recherche, publiés ou non, émanant des établissements d'enseignement et de recherche français ou étrangers, des laboratoires publics ou privés. 


\section{Éthique publique}

Revue internationale d'éthique sociétale et gouvernementale

vol. 20, n 2 | 2018

Les défis éthiques des déterminants sociaux de la santé

\section{«Dés-élitiser » la recherche pour favoriser l'équité en santé. Les recherches participatives avec des publics en situation de pauvreté en santé publique}

Christine Loignon, Sophie Dupéré, Baptiste Godrie et Caroline Leblanc

\section{(2) OpenEdition}

Journals

Édition électronique

URL : http://journals.openedition.org/ethiquepublique/4058

ISSN : 1929-7017

Éditeur

Éditions Nota bene

Ce document vous est offert par Bibliothèques de l'Université de Montréal

les bibliothèques/udem

Référence électronique

Christine Loignon, Sophie Dupéré, Baptiste Godrie et Caroline Leblanc, « « Dés-élitiser » la recherche pour favoriser l'équité en santé. Les recherches participatives avec des publics en situation de pauvreté en santé publique », Éthique publique [En ligne], vol. 20, n² 2 | 2018, mis en ligne le 02 avril 2019, consulté le 05 avril 2019. URL : http://journals.openedition.org/ethiquepublique/4058

Ce document a été généré automatiquement le 5 avril 2019.

Tous droits réservés 


\title{
« Dés-élitiser » la recherche pour favoriser l'équité en santé. Les recherches participatives avec des publics en situation de pauvreté en santé publique
}

\author{
Christine Loignon, Sophie Dupéré, Baptiste Godrie et Caroline Leblanc
}

1 De nombreux obstacles à l'accès aux services de santé et aux services sociaux pour les personnes en situation de pauvreté ont été documentés par des études canadiennes et québécoises. Dans notre projet ÉQUisanTÉ (Loignon et al., 2015a ; Loignon et al., 2017) et notre projet LIENSSS (Loignon et al., 2018), deux recherches participatives menées avec des personnes en situation de pauvreté, en collaboration avec des organisations de la communauté, nous avons pu mieux comprendre comment ces personnes font l'expérience des barrières géographiques, économiques, organisationnelles et socioculturelles dans le système de santé. Ces projets de recherche ont aussi permis d'impliquer les personnes dans la recherche de solutions pour remédier aux barrières d'accès aux soins et aux services de santé.

Dans le projet ÉQUIsanTÉ mené en partenariat avec ATD Quart Monde, les personnes en situation de pauvreté et les professionnels de la santé ont été impliqués dans toutes les étapes de la recherche de la définition de la question de recherche à la diffusion des résultats auprès de différentes instances, dont l'Organisation des Nations Unies (ONU) en 2015 et en 2018. Ces cochercheurs de la communauté ont identifié trois barrières qui nuisent à l'accès et à la qualité des soins des personnes en situation de pauvreté : 1) les conditions de vie des personnes en situation de pauvreté (état de salubrité et coût des logements, défis d'accès à la saine alimentation, etc.) ; 2) la faible qualité des interactions entre les professionnels de la santé et les personnes en situation de pauvreté ; 3) la complexité du système de soins de santé. La contribution de cette étude découle du fait que le devis de recherche participative favorisait un rôle actif des personnes en situation 
de pauvreté dans le déploiement des changements de pratiques dans les organisations locales de soins primaires, ce qui est rare (Loignon et al., 2017).

Dans le projet LIENSSS, mené en partenariat avec trois organismes d'alphabétisation, La Jarnigoine, Atout Lire et Tour de Lire, les personnes vivant avec un faible niveau de littératie (personnes analphabètes) ont exprimé à leur manière des préoccupations similaires. Elles ont expliqué avoir du mal à améliorer leur santé en raison de barrières d'accès aux services de santé, en déplorant notamment le temps réduit de consultation, le manque d'écoute des médecins, le coût pour prendre soin de sa santé, les " frais cachés » dans les cliniques, les difficultés de prise de rendez-vous en ligne. Elles ont également rapporté vivre de la stigmatisation et de la discrimination dans le système de santé du fait de leur niveau de littératie. Ces expériences ont nui à ce que Dixon-Woods et ses coauteurs appellent le phénomène de candidacy référant au sentiment de légitimité d'être reçu et accueilli dans le système de santé (Dixon-Woods et al., 2006).

4 De manière préoccupante, les personnes en situation de pauvreté et les membres de la communauté ayant participé à ces deux projets ont rapporté que l'accès ardu à la saine alimentation et l'environnement malsain participent aussi aux problèmes de santé et s'additionnent aux barrières rencontrées dans le système de soins de santé. Ces déterminants sociaux de la santé ont la particularité de s'inscrire dans leurs trajectoires de vie sans qu'aucun d'eux ne soit totalement détaché de l'autre. Cela nous renvoie à l'importance de penser la recherche en favorisant l'inclusion de la voix des personnes concernées directement par la pauvreté et l'exclusion sociale afin d'intégrer toute la complexité des situations vécues et que celle-ci se reflète dans les interventions de santé publique.

5 Les bénéfices de l'implication des personnes qui expérimentent au premier chef les barrières systémiques d'accès à la santé dans le développement de recherches et/ou d'interventions en santé sont documentés. Il est reconnu que les devis et les méthodes participatives renforcent le pouvoir d'agir des communautés (Eng et al., 2018) et permettent d'influencer plus efficacement les politiques de santé pour atteindre une meilleure équité (Cacari-Stone et al., 2018; Belone et al., 2017). Pourtant, peu de recherches au Canada interpellent activement les personnes marginalisées ou en situation de pauvreté (Montesanti et al., 2017). Ainsi, en impliquant des personnes en situation de pauvreté aux côtés de décideurs, par exemple dans un projet de recherche, il est possible de générer de nouvelles connaissances sur l'application des principes de partenariat en recherche (ex.: la participation équitable des membres de la communauté), une lacune majeure soulevée récemment par un groupe d'experts en recherche participative (Allen et al., 2017).

6 La participation des usagers est désormais inscrite dans les orientations stratégiques des établissements de santé et de services sociaux au Québec (MSSS, 2018). La coproduction des services et des interventions de santé avec ces personnes est présentée comme souhaitable dans ces documents, mais rarement explicitée ou mise en œuvre avec des effets documentés (Godrie, 2015 ; Montesanti et al., 2017). L'une des manières d'impliquer les usagers ou les membres de la communauté en situation de pauvreté dans l'élaboration des interventions en santé publique consiste à les amener à participer à la coconstruction des interventions au travers des dispositifs de recherche. Toutefois, force est de constater qu'une réflexion sur les enjeux éthiques et épistémologiques que cette coconstruction implique serait utile pour orienter les chercheurs et les membres de la communauté. 
7 En nous basant sur l'expérience et les données issues des processus participatifs de deux projets de recherche présentés précédemment, nous souhaitons aborder certains enjeux éthiques et épistémologiques de la participation (et de l'absence de participation) des personnes marginalisées à la recherche en santé publique. En nous inspirant des principes de la recherche communautaire participative ou engagée envers la communauté, nous proposerons quelques pistes pour "dés-élitiser » la recherche en santé publique. Avant tout, nous présentons un état de situation des inégalités sociales qui perdurent dans le système de santé québécois afin de justifier notre position épistémologique sur la participation des personnes exclues de la société à la production du savoir scientifique portant sur les interventions en santé les plus susceptibles de contribuer à améliorer leur situation.

\section{Un « système de santé malsain " pensé et réformé sans la voix des exclus}

8 Cette expression, "un système de santé malsain ", a été employée récemment par une personne en situation de pauvreté, membre d'ATD Quart Monde, qui participe à nos recherches devant un décideur de l'ONU à Montréal pour exprimer ses difficultés de navigation dans les soins de santé. Plus de $60 \%$ des Canadiens sont confrontés à des obstacles de navigation en santé, c'est-à-dire qu'ils éprouvent des difficultés majeures à obtenir des renseignements et des services de santé, à comprendre et à agir adéquatement en vue d'améliorer leur état de santé et à prendre eux-mêmes les décisions appropriées leur permettant d'améliorer leur santé (Ronson McNichol et Rootman, 2009). La navigation en santé est étroitement liée à la littératie en santé qui est elle-même un déterminant important de la santé étroitement lié aux autres déterminants sociaux de la santé au Canada (Conseil canadien de l'apprentissage, 2008). Une faible littératie en santé engendre des coûts importants pour les personnes, les communautés et les systèmes de santé (Mitic et Rootman, 2012; Poureslami et al., 2016). Elle nuit aux capacités des individus à surmonter les barrières structurelles d'accès à la santé liées à l'environnement bâti et à l'accès aux services de santé notamment (Kickbusch, 2001). De plus, une grande proportion de Canadiens ont un faible niveau de littératie, donc des difficultés majeures de lecture et d'écriture (Conseil canadien de l'apprentissage, 2008). Au Québec, la situation est préoccupante, car $53 \%$ des Québécois n'ont pas un niveau de littératie optimale pour bien fonctionner et $19 \%$ éprouvent de grandes difficultés en lecture et en écriture (ISQ, 2015).

Ces défis touchent en plus grande proportion les personnes qui vivent dans un contexte de défavorisation socioéconomique. C'est le cas notamment des personnes qui ont des emplois mal rémunérés ou qui bénéficient de l'aide sociale, qui ont de faibles niveaux de scolarité et maîtrisent peu l'anglais ou le français. Ces personnes sont les plus susceptibles de vivre des difficultés d'accès aux services dans le système de santé. Elles cumulent plusieurs conditions chroniques de santé tout en bénéficiant peu des interventions de santé publique et des soins primaires, ce qui les expose à une détérioration de leur santé et à une mortalité précoce (Loignon et al., 2015b).

Les interventions en santé publique, notamment en soins primaires de santé, peuvent contribuer à renforcer les barrières d'accès à la santé et participer à la stigmatisation des usagers et des membres de la communauté en situation de pauvreté, tout comme elles 
peuvent contribuer à améliorer leur accessibilité et leurs conditions de vie. Les interventions en santé (santé publique et soins et services sociaux) prennent rarement en considération les difficultés de navigation en santé des usagers et des membres de la communauté en situation de pauvreté, que l'on affuble parfois des termes " complexes » ou "difficiles à rejoindre ». Ces interventions intègrent peu les interactions qui existent entre un environnement bâti délétère ou favorable et les capacités des individus à accéder à la santé dans la communauté, incluant l'accès aux services de santé. De plus, ces interventions et les études sur les résultats de ces mêmes interventions intègrent peu la perspective des personnes marginalisées sur leurs défis de navigation en santé, car ces dernières sont difficiles à recruter et exclues de la plupart des études, notamment celles qui reposent sur les bases de données administratives en santé ou des questionnaires autoadministrés (Potvin, Moquet et Jones, 2010). Ceci est d'autant plus important que nos travaux ont révélé que les personnes en situation de pauvreté portent le fardeau et le regard réprobateur et vivent de la discrimination (enacted stigma) (Scambler, 2004) avec les professionnels du système de santé parce qu'elles n'arrivent simplement pas à répondre aux exigences de l'autogestion de la santé, prises dans la boucle d'une trajectoire de vie difficile et n'ayant pas accès à une alimentation ou à un logement sain.

11 Au Québec, des inégalités sociales de santé importantes persistent entre différents groupes sociaux (Boisvert, 2012; Hyppolite, 2012). Certains quartiers des villes de Montréal et de Québec, au Canada, présentent des taux de pauvreté dépassant le seuil de $40 \%$ retenu pour définir les zones de très forte concentration de la pauvreté (Séguin et al., 2017). L'espérance de vie des citoyens habitant les quartiers défavorisés est de onze ans inférieure à celle des citoyens habitant les quartiers favorisés (Agence de la santé et des services sociaux de Montréal, 2011). En 2016, une personne vivant seule qui recevait des prestations d'aide sociale disposait d'environ la moitié du revenu nécessaire pour couvrir ses besoins de base. Même les personnes qui recevaient des prestations du programme de Solidarité sociale - pourtant reconnues comme ayant des « contraintes à l'emploi » - ne percevaient pas un revenu suffisant pour sortir de la pauvreté (CEPE, 2017).

La situation est d'autant plus préoccupante qu'au Québec, la santé publique, les services sociaux et de santé de même que les systèmes d'éducation et d'aide sociale ont subi des réductions budgétaires majeures simultanées imposées par les gouvernements néolibéraux successifs. Ces politiques ont été décriées par de nombreux acteurs de santé publique pour être déconnectées des besoins et des réalités des populations et venir compromettre la santé des personnes ou des groupes sociaux les plus précaires de la société québécoise. La priorité est accordée au médico-hospitalier au détriment de la prévention, de la promotion de la santé, des services sociaux, des services adaptés aux personnes en situation de pauvreté des services à domicile et des organismes communautaires, et ce, malgré des évidences scientifiques solides sur les solutions pour contrer les inégalités sociales de recours aux soins et aux services notamment. Alors que nous assistons à l'effritement des lieux où les patients peuvent avoir un certain niveau d'influence sur le plan de la gouvernance en santé, la recherche en santé publique impliquant la participation des membres de la communauté s'avère une avenue pour améliorer l'équité en santé (De Koninck et al., 2018). 


\section{Enjeux éthiques de la participation ou de l'absence de la participation}

13 La recherche en santé publique, en particulier celle visant à déterminer et à réduire les inégalités de santé, peut retirer des retombées positives de la participation authentique et soutenue des membres de la communauté directement touchés par les inégalités sociales. Les effets positifs de la recherche participative ont été largement documentés ces dix dernières années à l'international : réductions des inégalités de santé, pratique réflexive, meilleure validité externe des résultats de recherche, recherche centrée sur les besoins culturels et sociaux des communautés, changement organisationnel, etc. (Wallerstein et al ., 2018; Bush et al., 2018 ; de las Nueces et al., 2012) Selon une récente revue systématique de littérature, $88 \%$ des projets impliquant des populations vulnérables ont eu les retombées positives suivantes: meilleurs résultats en termes de niveau de santé, de littératie en santé et d'accès aux services de santé (Cyril et al., 2015).

La littérature sur le sujet fait également état de deux enjeux éthiques majeurs concernant les recherches participatives: la participation accessoire des chercheurs sans formation professionnelle de recherche, qui peuvent dans certains cas se retrouver sans réel pouvoir décisionnel dans le processus de recherche; et la mise sur pied de projets désincarnés des besoins et des expertises des communautés sollicitées.

Les processus participatifs de recherche, des phases initiales de la recherche à la diffusion des résultats auprès des décideurs, offrent pourtant différentes possibilités d'impliquer les usagers et membres de la communauté vivant en situation de pauvreté dans l'élaboration des interventions en santé publique. Mais nous disposons de peu de données publiées sur ces processus, car certains chercheurs hésitent à publier sur les défis méthodologiques et éthiques rencontrés. Certains avancent que les conditions de production de savoirs scientifiques (biais de publication scientifique, barrières de financement, pression de performance scientifique, etc.) seraient en jeu, car la crédibilité des chercheurs pourrait être entachée (Lenette et al., 2019).

Dans ce contexte, il s'avère pertinent de réfléchir aux processus d'inclusion des citoyens et des communautés qui subissent les inégalités sociales dans les dispositifs de recherche qui se veulent porteurs de changements et de politiques publiques favorables à la santé et à l'équité. Cela dit, le mouvement mondial d'engagement en recherche des patients qui se déploie au Canada depuis 2014 sous l'égide de la Stratégie de recherche axée sur les patients, soutenue par les Instituts de recherche en santé du Canada et ailleurs dans le monde au travers d'INVOLVE (Angleterre) et de PCORI (États-Unis) s'est graduellement institutionnalisé ces dernières années au sein des instances universitaires et des organismes subventionnaires contribuant à la recherche en santé. Au Québec, ce développement s'est surtout déployé par des projets de recherche sur les soins de santé en impliquant des patients " privilégiés » ayant une condition de santé ou une maladie et pour lesquels on exige qu'ils soient "partenaires» de leurs soins, la plupart étant éduqués et/ou aisés et surtout bénéficiant d'un accès aux soins. Peu de remise en question a eu lieu à propos du niveau de participation des "non-patients ", ces usagers qui ne bénéficient pas des soins ou qui ont un rapport complexe à la santé ou à la maladie en raison de leurs conditions sociales. Nous pensons que cela pave la voie à un débat sur la contribution des acteurs et des chercheurs en santé publique impliqués avec ces usagers dans des projets de recherche pour réduire les inégalités sociales de santé. 
Certains de ces acteurs et chercheurs se sont engagés ou s'engagent dans ce mouvement notamment par des recherches communautaires participatives. En revanche, il existe peu de ponts ou d'échanges d'expertises entre la recherche et l'intervention sur les soins primaires et la recherche et l'intervention sur les déterminants sociaux de la santé (que l'on oppose souvent d'ailleurs) et surtout, il existe peu de structures qui soutiennent les capacités et le financement de la recherche en santé publique, impliquant la participation des usagers et membres de la communauté subissant les inégalités sociales.

La recherche engagée envers la communauté, la recherche participative communautaire ou la recherche-action participative sont des devis reconnus mondialement pour favoriser l'engagement en recherche des membres de la communauté afin de réduire les inégalités sociales de santé et contribuer ainsi au changement social. (Wallerstein, 2018; Cyril et al., 2015). Malgré le fait que plusieurs chercheurs mettent en avant de tels dispositifs de recherche pour influencer les interventions et les politiques publiques favorables en santé, nous disposons encore de peu de connaissances scientifiques sur ce type de recherche engagée envers la communauté. Il y a une utilisation un peu abusive du terme recherche participative que l'on utilise à toutes les sauces. Nous trouvons une telle diversité d'approches de recherche participative ou partenariale avec des degrés forts différents d'engagement des personnes, des valeurs et des finalités fort différentes, si bien qu'il est difficile d'apprécier les effets de la recherche participative, les dispositifs et les méthodes optimales. D'ailleurs, des revues récentes ont constaté que les recherches dites participatives sont en fait davantage de nature consultative que réellement participative (Tierney, et al., 2016). Certains membres du public seraient condamnés à n'être considérés que des publics « faibles » (weak public) et ainsi à n'être que peu présents en recherche ou n'avoir que peu d'influence auprès des chercheurs ou des décideurs (Gibson, Welsman et Britten, 2016).

Une recherche engagée devrait permettre une participation active, équitable et réelle des personnes pour éviter leur instrumentalisation et permettre l'expression et la prise en compte de la diversité de leurs points de vue et savoirs (Carrel, 2017). McCoy dénonce à juste titre que la participation du public à la recherche implique souvent les personnes jugées les plus compétentes et disposées à participer à la recherche en raison de leur scolarité (les personnes titulaires d'un diplôme universitaire, notamment), de leur situation financière (le fait de pouvoir avancer les dépenses associées à leur participation) et du contexte (avoir accès à un transport, par exemple) (2018). Pour ces raisons, certains chercheurs évoquent le risque de participation accessoire ou symbolique (tokenistic) dans les projets qui impliquent les personnes membres du public ou de la communauté. (Domeck, 2014; Charlebois et al., 2014). D'autres chercheurs soulignent que la participation authentique et qui répond aux attentes des partenaires de la communauté est également menacée par les contraintes des institutions universitaires et des exigences des organismes subventionnaires. À titre d'exemple, les critères de rédaction des demandes et la durée des financements ne seraient pas adaptés respectivement au contexte des projets de recherche en partenariat et au temps nécessaire au développement d'un partenariat avec les membres du public (Young et Wabano, 2018; Dupéré et al., 2017). 


\section{Dés-élitiser la recherche en santé publique : une piste épistémologique}

19 La recherche participative ou engagée envers la communauté repose sur la prémisse que la production des savoirs scientifiques ne doit pas être l'apanage unique de chercheurs universitaires (Wallerstein, 2018). Elle doit permettre aux membres de la société qui ont une histoire de discrimination ou de colonisation de s'affranchir de ces processus d'exclusion sociale, et de participer activement à la recherche pour transformer leur communauté et améliorer l'équité sociale. Ce processus est incarné par le concept de décolonisation de la recherche (decolonize research) introduit par Smith (1999). Cette chercheure a contribué à infléchir un positionnement idéologique chez ses pairs en ce qui trait au rôle de la science dans la société. Ce concept implique de mener des recherches en respectant les valeurs et les coutumes des peuples autochtones et en évitant l'instrumentalisation (Wallerstein, 2018: 355). Plus radicalement, ce concept est porteur d'une recherche qui se fait en collaboration avec les membres de la communauté et donc d'une recherche engagée envers sa ou ses communautés, en partant de leurs besoins et de leurs préoccupations et non de ceux des chercheurs ou des décideurs en santé publique. Ce cas de figure, comme nous l'avons vu précédemment, apparaît rarissime.

20 Le terme de décolonisation de la recherche renvoie donc à un ensemble de valeurs, comme la propriété des données par les collectivités partenaires des projets de recherche, et de méthodologie pour co-interpréter les données ou les diffuser de manière conjointe. Ce terme inclut aussi la possibilité, le droit au désaccord sur l'interprétation des données de recherche, qui permet aux chercheurs de conserver leur liberté universitaire et aux communautés de préserver leur droit à l'interprétation. Dans ce cas, les publications visent à faire état des désaccords d'interprétation plutôt qu'à les gommer de manière artificielle pour faire prévaloir uniquement le point de vue des universitaires (Schnarch et FNCNAHO, 2004).

21 Puisque le terme de décolonisation a été forgé pour désigner la réalité de la recherche participative avec les communautés autochtones, nous choisissons quant à nous de parler de processus de «dés-élitisation» de la recherche. Ce processus renvoie à différentes dimensions, notamment celle de recourir aux méthodes adaptées aux intérêts, aux valeurs et aux capacités des personnes ainsi qu'aux méthodes qui ont été développées par les communautés pour réfléchir sur leurs conditions de vie et de santé. Ces méthodes telles que le croisement de savoirs, une méthode développée par le mouvement ATD Quart Monde, doivent se tailler une place dans la recherche (De Laat, 2016).

Nous avons eu recours au croisement des savoirs durant le projet ÉQUIsanTÉ. Cette méthode met en place les conditions nécessaires de la rencontre et du dialogue entre trois sources de savoir: les savoirs universitaires et théoriques, les savoirs d'action et d'engagement et les savoirs expérientiels. Un des principes fondamentaux du croisement des savoirs est de permettre un travail continu de réflexion et de co-construction de savoirs en groupes de pairs pour ensuite produire un résultat commun. Cela permet d'amenuiser les rapports inégalitaires qui peuvent souvent survenir lorsque la parole des personnes défavorisées ou marginalisées est, par exemple, moins valorisée dans l'espace public ou lorsque les personnes sont moins habituées à prendre la parole devant d'autres personnes au statut social privilégié (Carrel, 2017). Cette méthode a eu des retombées multiples que nous avons décrites ailleurs (Loignon et al., 2017). 
res chercheurs ont souligné que l'inclusion des populations marginalisées dans un processus de recherche permettait d'obtenir des données plus pertinentes, d'améliorer la validité des instruments de recherche et de tester la faisabilité de l'étude pour que les données soient viables dans le «monde réel » (Sheridan et al., 2017). Par contre, il peut s'avérer difficile de croiser les différents savoirs et d'opter pour une méthode participative en recherche. L'écart entre les statuts socioculturel, économique et politique peut engendrer des tensions lorsqu'on amène différents acteurs à interagir. Les relations de pouvoir liées à la classe sociale peuvent devenir un enjeu marquant. Il peut être complexe de concilier les besoins des chercheurs avec ceux des "participants cochercheurs" ou ceux des membres de la communauté à la recherche (Sheridan et al., 2017). Il n'est pas rare que les membres de la communauté se sentent impressionnés par le statut des professionnels et des décideurs, et qu'ils prétendent devoir être formés pour être entendus (Favre, Lainard et Loiseau, 2012). C'est dans cette optique qu'il faut considérer la participation active des membres de la communauté comme levier afin de réduire la distance sociale et créer une rencontre qui permet de comprendre ou de réunir ces deux types d'acteurs qui ont des logiques ou des visions du monde différentes.

On ne peut résoudre les problèmes d'une population concernée sans qu'elle prenne en main elle-même son sort, sans, au minimum, qu'elle soit étroitement associée à tous les stades de l'action, faute de quoi les solutions recherchées risquent bien de ne pas modifier en profondeur les causes qui engendrent les situations que l'on veut transformer (Tilman, 2004 : 33).

er les moyens aux personnes en situation de pauvreté de se sentir reconnues est nécessaire pour lutter contre la grande pauvreté et l'exclusion sociale (DeLaat et al., 2018). Lorsque l'on se base sur l'approche AVEC développée par le collectif VAATAVEC, il faut prendre le temps de reconnaitre les rapports de pouvoir, de comprendre les différentes cultures et logiques en présence, de les reconnaître et de les respecter et finalement d'établir des stratégies, notamment lors des prises de décision pour tendre vers une plus grande égalité (Collectif VAATAVEC, 2014). Pour y parvenir, il faut créer des espaces de réflexion et de dialogue qui permettent à toutes et tous de partager leur pensée sans censure et sans jugement. Les espaces en non-mixité constituent une stratégie intéressante (Dupéré et al., 2017). En d'autres mots, mettre en place un environnement avec des conditions gagnantes pour maximiser leur intérêt et leur engagement dans la recherche en les incluant à la préparation des réunions et des présentations, en leur apportant un soutien pour favoriser leur implication et en fournissant une rétroaction constante des démarches (Sheridan et al., 2017 ; Dupéré et al., 2017).

Nous argumentons qu'il est important de « dés-élitiser » la recherche et de permettre aux organisations engagées dans la lutte contre la pauvreté ou l'exclusion sociale, mais surtout aux personnes qui subissent cette pauvreté et qui sont exclues de la recherche de prendre leur place dans la recherche. Plusieurs défis se posent dans le processus d'engagement en recherche des personnes qui ne savent pas lire ou écrire, qui ont une déficience intellectuelle ou physique ou encore celles qui vivent avec des dépendances ou des problèmes de santé mentale graves. Cependant, nous arguons à partir de nos expériences des devis participatifs et de la littérature existante que ces défis peuvent être surmontés. En outre, nous proposons de recourir aux méthodes visuelles, des méthodes adaptées qui permettent de réduire les hiérarchies de savoirs qui prévalent entre les chercheurs universitaires, les cliniciens, les décideurs et les membres de la communauté. Les méthodes visuelles participatives, que nous avons expérimentées dans ÉQUIsanTÉ et LIENSSS, génèrent des données contextuelles riches et de bonne qualité, et cela est 
particulièrement important lorsqu'il s'agit de personnes à faible niveau de littératie (Greyson et al., 2017). Les autres raisons parmi les plus courantes justifiant le recours à ces méthodes sont les suivantes : 1) rendre la recherche ouverte aux enjeux des milieux de pratique et aux perspectives des usagers ; 2) combler l'écart entre la diffusion de résultats de recherche et la valorisation de connaissances par les décideurs de politique et les milieux de pratiques (D’Amico et al., 2016) ; 3) améliorer l'équité en matière de santé tout en renforçant les capacités de collaboration. Les connaissances acquises grâce à de tels projets de recherche proviennent des patients plutôt que des professionnels de la santé, ce qui reflète les expériences vécues des individus ou des communautés (D’Amico et al., 2016).

Nous avons eu l'occasion de recourir aux méthodes visuelles pour faciliter et soutenir cette participation active en recherche de personnes issues d'organisations communautaires luttant contre la pauvreté ou pour l'alphabétisation. L'utilisation de méthodes visuelles dans le processus de recherche (et non comme moyen de diffusion des résultats) est relativement récente dans la recherche en santé publique. Nous avons été confrontés en tant que chercheurs à de nombreux enjeux éthiques liés au recours aux photos, mais surtout aux vidéos dans le cadre du projet LIENSSS qui reposait sur la méthode Digital Story Telling. Même si nous avons documenté les avantages de ces méthodes, tout comme d'autres l'ont fait, nous avons pu constater combien le jugement et la bienveillance des chercheurs universitaires et issus de la communauté sont de mise à tout moment. Par exemple, les vidéos produites dans le cadre de notre projet pouvaient, lorsque présentées sans les personnes ayant participé au projet ou sans contextualisation du projet, contribuer à stigmatiser davantage ces personnes.

Le recours aux méthodes visuelles demande une vigilance accrue (Cox, 2014). Il peut être problématique d'assurer l'anonymat des personnes participant à des études utilisant des méthodes visuelles. Souvent, elles souhaitent rendre leur histoire publique, mais elles pourraient avoir des regrets plus tard à ce sujet. Prenons l'exemple de personnes vivant dans une petite ou moyenne localité qui, dans le cadre d'une étude, expliqueraient leurs stratagèmes pour cacher leurs difficultés en lecture et en écriture dans le but de paraître fonctionnelles à cet égard. Présenter leur histoire publiquement pourrait leur causer des torts ultérieurement. Il faut bien les renseigner à ce sujet et leur offrir différentes options, notamment la possibilité de rendre leur visage flou ou de le masquer d'un trait noir pour le rendre méconnaissable, et utiliser un pseudonyme.

Enfin, mentionnons que «dés-élitiser» la recherche passe par une posture épistémologique qui évite la hiérarchisation des savoirs, où les savoirs universitaires ne dominent pas les savoirs expérientiels, et qui soutient des approches participatives favorisant les rapports égalitaires au sein des projets, ce qui suppose une flexibilité dans les méthodes de recherche qui doivent être adaptées aux contextes et aux valeurs des personnes issues de la communauté.

\section{Conclusion}

Les interventions en santé publique, notamment celles qui relèvent du champ d'interventions en soins primaires et communautaires de santé, peuvent contribuer à réduire ou à aggraver les inégalités sociales de santé. Il est reconnu que ces interventions se sont surtout développées sans la participation des usagers et membres de la communauté en situation de pauvreté, tant sur le plan de leur conception que de leur 
mise en œuvre, ce qui concourt au fait que plusieurs interventions ne bénéficient pas à ceux-ci. De plus, ces usagers et membres de la communauté ne participent pas ou peu à la gouvernance du système de santé. Pourtant, comme nous l'avons soulevé, la pauvreté et les inégalités entre les groupes sociaux au Québec ne sont pas des phénomènes marginaux. Les déterminants sociaux de santé tels que la faible littératie et les barrières d'accès aux soins et aux services de santé concernent une large proportion de la population du Québec à différents niveaux. Mais surtout les déterminants sociaux interagissent entre eux et empêchent les personnes en situation de pauvreté d'améliorer leur santé. Comme nous l'avons explicité dans le présent article, la recherche engagée envers la communauté s'avère un levier pour améliorer l'équité en santé mais elle comprend de nombreux défis.

Historiquement, pour contourner ces défis, certains chercheurs et acteurs de la santé publique se sont engagés dans des projets de recherches communautaires participatives avec les membres de la population subissant de la discrimination, de la marginalisation ou des inégalités sociales de santé. Ce que nous avons argumenté dans cet article c'est que compte tenu de l'institutionnalisation de la recherche en partenariat avec les usagers et membres du public qui a cours, il faut s'interroger sur les enjeux éthiques et épistémologiques des méthodes pour favoriser la participation en recherche des personnes en situation de pauvreté et membres de la communauté marginalisés. Nous en avons soulevé plusieurs et nous avons proposé quelques avenues de solutions, comme celle de recourir à des méthodes adaptées telles que les méthodes visuelles et de soutenir une approche qui vise à " dés-élitiser » la recherche en santé publique.

31 Il est important de soulever que « dés-élitiser » la recherche implique un mouvement de réciprocité soutenue entre, d'une part, le milieu universitaire, notamment les universités, mais aussi les organismes subventionnaires et, d'autre part, le milieu communautaire, ses organisations et ses citoyens mobilisés. Le milieu universitaire peut s'engager à outiller et à habiliter les membres de la communauté à développer leurs compétences et leurs expertises pour participer activement à la recherche. Le milieu communautaire peut, quant à lui, s'engager à partager ses expertises et ses méthodes pour faciliter la participation des membres qui ne sont pas formés en recherche, mais qui détiennent une expertise unique. Enfin, nous arguons que sans modalités structurelles (financement subventionnaire, laboratoire de recherche, formation universitaire, etc.) ce processus de "dés-élitisation" est voué à demeurer marginal, ce qui ne fera que perpétuer les hiérarchies des savoirs et les inégalités sociales en santé publique.

\section{BIBLIOGRAPHIE}

AGENCE DE LA SANTÉ ET DES SERVICES SOCIAUX DE MONTRÉAL (2011), Rapport du directeur de santé publique 2011 - Les inégalités sociales de santé à Montréal : le chemin parcouru, $2^{\mathrm{e}}$ édition, [en ligne], https:// santemontreal.qc.ca/fileadmin/fichiers/professionnels/DRSP/Directeur/Rapports/ Rapport_ISS-2011.pdf (consulté le 31 mars 2019). 
ALLEN, Michele L. et al. (2017), « Engaging with communities, engaging with patients: amendment to NAPCRG 1998 Policy Statement on Responsible Research with Communities », Family Practice, vol. 34, $\mathrm{n}^{\circ}$ 3, p. 313-321.

BELONE, L., et al. (2017), « Community based participatory research principles and strategies for co-creating culturally-centered interventions with Native communities: A partnership between the University of New Mexico and the Pueblo of Jemez with implications for other ethno-cultural communities ", dans N. Zane, F. Leong et G. Bernal (dir.), Evidence-Based Psychological Practice with Ethnic Minorities: Culturally Informed Research and Clinical Strategies, p. 199-220.

BOISVERT, Raymond (2012), Les inégalités sociales de santé ne sont pas une fatalité: voyons-y!, Rapport du directeur de santé publique sur les inégalités sociales de santé en Mauricie et au Centre-duQuébec, Direction de la santé publique de la Mauricie et du Centre-du-Québec.

CACARI-STONE, Lisa et al. (2018), « The Role of Community-Based Participatory Research in Policy Advocacy ", dans Nina Wallerstein et al., CBPR for Health: Advancing Social and Health Equity, $3^{\mathrm{e}}$ edition, San Francisco, Jossey-Bass.

CARREL, Marion et al. (2017), « Vers une épistémologie post-pauvreté? Les enjeux du croisement des savoirs entre personnes en situation de pauvreté, patricien(ne)s et universitaires. Retours sur la recherche ÉQUIsanTÉ au Québec ", Sociologie et Sociétés, vol. 49, nº 1.

CEPE - CENTRE D'ÉTUDE SUR LA PAUVRETÉ ET L'EXCLUSION SOCIALE (2017), La pauvreté, les inégalités et l'exclusion au Québec : état de situation en 2016, [en ligne], https://www.mess.gouv.qc.ca/ publications/pdf/CEPE_Etat_Situation_2016.pdf (consulté le 331 mars 2019).

CHARLEBOIS, Katleen et al. (2014), « L'implication des personnes vulnérables dans la recherche participative en soins de santé primaires : une revue de la littérature », Global Health Promotion, vol. $21, \mathrm{n}^{\circ} 3$, p. 38-45

COLLECTIF VAATVEC (2014), L'AVEC, pour faire ensemble. Un guide de pratiques, de réflexions et d'outils, Québec, FRQSC (Programme Actions concertées).

CONSEIL CANADIEN DE L'APPRENTISSAGE (2008), La littératie en santé au Canada: une question de bien-être. Ottawa, Canada: Conseil canadien de l'apprentissage, [en ligne], http://www.bdaa.ca/biblio/ recherche/cca/sante/sante.pdf (consulté le 28 février 2018).

cox, Susan et al. (2014), Guidelines for Ethical Visual Research Methods, Melbourne School of Population and Global Health, The University of Melbourne.

CYRIL, Sheila, et al. (2015), « Exploring the role of community engagement in improving the health of disadvantaged populations: A systematic review », Global Health Action, vol. 8, p. 1-12.

D'AMIco, Miranda et al. (2016), « Research as intervention? Exploring the health and well-being of children and youth facing global adversity through participatory visual methods ", Global Public Health, vol. 11, n 5/6, p. 528-545.

DE KONINCK, Maria et al. (2018), Remettre la santé et la démocratie au cour de notre système de santé, [en ligne], http://www.cssante.com/sites/www.cssante.com/files/180710_sante_et_democratie.pdf (consulté le 6 octobre 2018).

DE LAAT, Marianne et al. (2016), « Le croisement des savoirs et des pratiques avec des personnes en situation de pauvreté : Une condition nécessaire pour une société du savoir inclusive », Globe, Revue internationale d'études québécoises, vol. 17, nº 2, p. 69-90.

DIXON-WOODS, Mary et al. (2006), « Conducting a critical interpretive synthesis of the literature on access to healthcare by vulnerable groups », BMC Medical Research Methodology, vol. 6, nº 1, 35. 
DOMECQ, Juan Pablo et al. (2014), Patient engagement in research: a systematic review, BMC Health Services Research, vol. 14, n 89, p. 1-9.

DUPÉRÉ, Sophie et al. (2017), Supporting participation of marginalisd people in community-beased participatory action research: challenges, struggles and key lessons, QHR Conference, Québec.

FAVRE, Marie, Marylise LAINARD et Laura LoISEAU (2012), « Participation des usagers dans les projets de santé publique : réalités et paradoxes ", Spécificités, vol. 5, nº1, p. 139-152.

ENG, Eugenia et al. (2018), « Partnership, Transparency, and Accountability: Changing Systems to Enhance Racial Equity in Cancer Care and Outcomes ", dans Nina Wallerstein et al. (dir.), Community Based Participatory Research for Health: Advancing Social and Health Equity, $3^{\mathrm{e}}$ édition, San Francisco, Jossey-Bass, p. 107-122.

GIBSON, Andy, Jo WELSMAN et Nicky BRITTEN (2016), « Evaluating patient and public involvement in research: from theoretical model to practical workshop ", Health Expectations, vol. 20, n 5, p. 826-835.

GODRIE, Baptiste (2015), « La participation publique favorise-t-elle l'empowerment ? Un état des lieux au Québec et dans le monde anglo-saxon », Sciences et actions sociales, [en ligne], http:// www.sas-revue.org/index.php/12-dossiers/17-la-participation-publique-favorise-t-elle-lempowerment-un-etat-des-lieux-au-quebec-et-dans-le-monde-anglo-saxon (consulté le 12 novembre 2019).

GREYSON, Devon, Heather O'BRIEN, et Jean SHOVELLER (2017), « Information world mapping: A participatory arts-based elicitation method for information behavior interviews », Library \& Information Science Research, vol. 39, $\mathrm{n}^{\circ}$ 2, p. 149-157.

HYPPOLITE, Shelley-Rose (2012), Comprendre et agir autrement pour viser l'équité en santé dans la région de la Capitale-Nationale, Rapport du directeur régional de santé publique sur les inégalités sociales de santé, Agence de la santé et des services sociaux de la Capitale-Nationale

ISQ - INSTITUT DE LA STATISTIQUE DU QUÉBEC (2015), Rapport québécois du Programme d'évaluation internationale des compétences des adultes (PEICA) de l'OCDE, [en ligne], http://www.stat.gouv.qc.ca/ statistiques/education/alphabetisation-litteratie/peica.pdf (consulté le 31 mars 2019).

KICKBUSCH, Ilona (2001), « Health literacy: Addressing the health and education divide », Health Promotion International, vol. 16, $\mathrm{n}^{\circ}$ 3, p. 289-297.

LENETTE, Caroline et al. (à paraître), « Brushed under carpet: Examining the complexities of participatory research », Research for All, sous presse.

LOIGNON, Christine et al. (2015a), « Perceived barriers to responsive care for persons living in poverty in Quebec », Canada, the EQUIhealhTY project, International Journal for Equity and Health, vol. $14, \mathrm{n}^{\circ} 1$.

LOIGNON, Christine et al. (2015b), « Providing care to vulnerable populations: A qualitative study among GPs working in deprived areas in Montreal ", Canada, Family Practice, vol. 32, n 2, p. 232-236.

LOIGNON, Chirstine et al. (2018), « Health literacy - Engaging the community in the co-creation of meaningful health navigation services: A study protocol », BMC Health Services Research, [en ligne], https://www.ncbi.nlm.nih.gov/pmc/articles/PMC6022349/ (consulté le 31 mars 2019).

LOIGNON, Christine et al. (2017), « Le projet ÉQUIsanTÉ : une expérience de valorisation des savoirs expérientiels à visée réflexive et transformatrice ", dans Sébastien Carrier et al. (dir.), 
L'engagement de la personne dans les soins de santé et services sociaux - Regards croisés France/Québec, Québec, Presses de l'Université du Québec.

MCCOY, Matthew S. et al. (2018), « National standards for Public Involvement in Research: missing the forest for the trees ", Journal of Medical Ethics, vol. 44, $\mathrm{n}^{\circ} 12$, p. 801-804.

MSSS - MINISTÈRE DE LA SANTÉ ET DES SERVICES SOCIAUX (2018), Cadre de référence de l'approche de partenariat entre les usagers, leurs proches et les acteurs en santé et services sociaux, [en ligne], http:// publications.msss.gouv.qc.ca/msss/fichiers/2018/18-727-01W.pdf (consulté le 12 novembre 2018).

MITIC, Wayne et Irving ROOTMAN (2012), Une approche intersectorielle pour améliorer la littératie en santé des Canadiens et Canadiennes, Document de travail, Public Health Association of BC, [en ligne], http://www.phabc.org/userfiles/file/ActionPlantoImproveHealthLiteracyinCanada_FRENCH.pdf (consulté le 28 février 2018).

MONTESANTI, Stephanie et al. (2017) « Enabling the participation of marginalized populations: a case studies from a health care organization in Ontario », Health Promotion International, no 32, p. 636-649.

POTVIN, Louise, Marie-José MOQUET et Catherine M. JONEs (2010), « La réduction des inégalités: un objectif prioritaire des systèmes de santé ", Réduire les inégalités sociales en santé, Saint-Denis, INPES, coll. Santé en action, p. 52-61.

POURESLAMI, Iraj et al. (2016), « Health literacy and chronic disease management: drawing from expert knowledge to set an agenda », Health Promotion International, vol. 32, n 4, p. 743-754. RONSON MCNICHOL, Barbara et Irving ROOTMAN (2009), « Literacy and health literacy: New understandings about their impact on health », dans D. Raphael, Social determinants of health : Canadian perspectives, 2e édition, Toronto, Canadian Scholar's Press, p. 170-185.

SCAMBLER, Graham (2004), « Re-framing Stigma: Felt and enacted stigm and challenges to the sociology of chronic and disabling conditions ", Social Theory and Health, vol. 2, n 1, p. 29-46. SCHNARCH, Brian et FIRST NATIONS CENTRE NATIONAL ABORIGINAL HEALTH ORGANIZATION (2004), « Ownership, Control, Access, and Possession (OCAP) or Self-Determination Applied to Research. A Critical Analysis of Contemporary First Nations Research and Some Options for First Nations Communities ", Journal of Aboriginal Health, p. 80-95.

SÉGUIN, Anne-Marie et Philippe APPARICIO (2017), « Poverty and wealth in the post-war Montreal area: A landscape in motion », dans D. Fougères et R. MacLeod (dir.), Montreal: The History of a North American City, Montréal, McGill-Queen's University Press.

SHERIDAN, Susan et al. (2017), « The PCORI Engagement Rubric: Promising Practices for Partnering in Research », Annals of Family Medicine, vol. 15, nº 2, p. 165-170.

SMITH, Linda Tuhiwai (1999), Decolonizing methodologies: Research and Indigenous peoples, Dunedin, University of Otago Press.

TILMAN, Francis (2004), Penser le projet : Concepts et outils d'une pédagogie émancipatrice, Lyon, Chronique sociale Éditions.

TIERNEY, Edel et al. (2016), « A critical analysis of the implementation of service user involvement in primary care research and health service development using normalization process theory ", Health Expectations, vol. 19, $\mathrm{n}^{\circ}$ 3, p. 501-515.

WALLERSTEIN, Nina et al. (2018), Community Based Participatory Research for Health: Advancing Social and Health Equity, $3^{\mathrm{e}}$ edition, San Francisco, Jossey-Bass 
YOUNG, Nancy L. et Mary Jo WABANo (2018), « Beyond the patient: lessons from community

engagement in a rural First Nation », CMAJ, $\mathrm{n}^{\circ}$ 190, (Suppl. 1), p. S16-S18.

\section{RÉSUMÉS}

La participation en recherche des citoyens et des membres des communautés concernés directement par les inégalités sociales et l'exclusion s'avère un levier pour favoriser l'équité en santé. Or elle est peu débattue dans l'étude des déterminants sociaux de la santé et dans le champ de la santé publique. Pourtant, il y a des enjeux éthiques et épistémologiques à la participation des personnes exclues et stigmatisées à la recherche en santé publique, notamment dans le contexte des soins primaires. Dans le présent article, nous proposons une réflexion critique et nuancée nourrie par une expertise de recherche engagée dans la communauté développée à partir de deux projets de recherches récents menés avec des personnes en situation de pauvreté. Ce texte se veut un plaidoyer en faveur de la participation des personnes marginalisées à la science pour inscrire l'équité en santé au calendrier des politiques publiques.

Research involving citizens and community members who are most affected by social inequality and exclusion is a powerful tool to promote health equity. However, diverse ethical and epistemological challenges emerge from public participation in public health research, particularly when it comes to individuals who are stigmatized and marginalized in primary health care settings. Indeed, there has been little discussion on the public participation in studies pertaining to the social determinants of health. In this article, we offer a critical and nuanced reflection on the subject, drawing on expertise developed throughout twor recent research studies conducted with people living in poverty. This text makes the case for increasing participation of marginalized persons in scientific research, allowing for the inclusion of health equity on the public policies agenda.

INDEX

Keywords : participatory research, social inequalities, social vulnerability, exclusion, poverty, ethical issues, epistemology

Mots-clés : recherches participatives, inégalités sociales, vulnérabilité sociale, exclusion, pauvreté, enjeux éthiques, épistémologie

\section{AUTEURS}

\section{CHRISTINE LOIGNON}

Christine Loignon est professeure agrégée à l'Université de Sherbrooke depuis 2010. Elle est chercheure régulière au Centre de recherche sur les innovations en santé et chercheure associée à l'Institut national de santé publique du Québec. Elle possède une formation multidisciplinaire en sciences sociales (sciences politiques et sociologie) et détient un Ph. D. en santé publique. Sociologue de la santé œuvrant en recherche appliquée au système de santé, elle mène plusieurs projets de recherche participative avec les populations marginalisées et les professionnels de la santé. 


\section{SOPHIE DUPÉRÉ}

Sophie Dupéré est professeure agrégée à la Faculté des sciences infirmières de l'Université Laval et membre du Centre de recherche sur les inégalités sociales et les discriminations (CRÉMIS). Elle détient une expertise en santé communautaire et en promotion de la santé. Ses projets de recherche tentent de mieux comprendre les réalités des personnes en situation de pauvreté et de marginalité et d'améliorer les pratiques, les programmes, les services et les politiques les concernant. Elle a notamment mené des recherches sur l'accès aux soins et l'équité en santé. Elle a participé à titre de chercheure principale ou de cochercheure à une quinzaine projets de recherche-action participative, menés avec diverses organisations issues de divers milieux et secteurs (communautaire, municipal, milieu de santé et services sociaux, milieu de l'éducation).

\section{BAPTISTE GODRIE}

Baptiste Godrie est sociologue au Centre de recherche de Montréal sur les inégalités sociales, les discriminations et les pratiques alternatives de citoyenneté (CREMIS, Centre intégré universitaire de santé et de services sociaux du Centre-Sud-de-l'île-de-Montréal) et professeur associé au Département de sociologie de l'Université de Montréal. Il codirige le groupe de travail 21 Diversité des savoirs de l'Association internationale des sociologues de langue française et le champ thématique Savoirs du CREMIS. Ses recherches portent sur les inégalités sociales, la participation sociale et la production des savoirs.

\section{CAROLINE LEBLANC}

Caroline Leblanc détient une maîtrise en travail social. Elle est professionnelle de recherche au Centre de recherche Charles Lemoyne-Sagnenay sur les innovations en santé, co-responsable de l'initiative Patients-Partenaires ainsi que chargée de cours à l'Université de Sherbrooke dans un programme qui s'adresse aux usagers afin qu'ils développent des compétences pour contribuer à la formation en travail social. Caroline est une personne engagée auprès des populations exclues de notre société. L'inclusion, la réduction des inégalités sociales et la dignité humaine sont au cœur de ses intérêts de recherche. Pour elle, il est important d'impliquer les personnes concernées par les problématiques sociales comme partie prenante dans la réponse à leurs besoins. 